\title{
Progress in Physics
}

\section{Tributes to Max Planck}

$\mathrm{T}$ HE recent issue of the Annalen der Physik has been dedicated to Prof. Max Planck in honour of his eightieth birthday on April 23 (see Nature, April 23, p. 720). Some of the twenty-two papers offered to him by his friends, colleagues and pupils may be picked out for mention here.

(1) The first paper is by N. Bohr on the quantum of action and atomic nuclei. Bohr contrasts the situation in the early days of interpretation of atomic spectra with the present situation of nuclear theory. In the atom, the weak and long-range Coulomb forces between the particles made possible the successful application of the correspondence principle. This failed only at an advanced stage of interpretation, when it was superseded by a fundamental revision of the laws governing observation in physics.

The nucleus, on the other hand, is held together by strong short-range forces with saturation properties, which couple the constituent parts to form an indivisible system. Gamow's theory of $\alpha$-ray spectra gave an indication of the energy levels of this system. These cannot be explained on the assumption of individual particles moving in a fixed field of force as of the Coulomb type. The strong coupling of the particles and easy transfer of energy between them is revealed in the transformation processes of nuclei under neutron bombardment.

Fermi showed that the impinging energy of the neutron is spread over the whole nucleus, with formation of an unstable nucleus. This strong coupling thus makes a nucleus similar to an elastic system in dynamics, in which the effect of a shock will be distributed over a great number of proper frequencies forming an extended and finely graded spectrum. The energy of the bombarding particle 'heats' the nucleus and an 'evaporation' of some of the nuclear constituents may follow this process (cf. NATURE, 137, 344 and 351 (1936).

(2) In the second paper, W. Heisenberg discusses the relevancy of the length $r_{0}=e^{2} / m c^{2}=2 \cdot 81 \times 10^{-15}$ cm., which he considers to be one of the 'primary universal constants'. By this term are designated universal constants implying some kind of invariance of the physical laws. Examples are $c$, determining the Lorentz-invariance and $h$, determining the interchange relations and the invariance of the quantum laws against rotation in the Hilbert space. These 'primary universal constants' indicate the limits where the laws formulated for our initial domain of experience break down $(v \approx c, E \approx h \nu)$. The length $r_{0}$ may be taken to indicate the limit down to but not beyond which the usual quantum mechanics hold. The new laws governing physics at distances less than $r_{0}$ may be expected to manifest themselves in the following phenomena :

(a) Self-energy and radius of the electron, Sommerfeld's fine structure constant, $\alpha=e^{2} / h c$. (Since $e<\sqrt{h c}$, classical explanations for these are not to be expected.)

(b) $\beta$-Disintegration. The present difficulties arise from imposing arbitrary limits on certain integrations ; this treatment may be considered as a kind of correspondence to the treatment taking account of the new laws below $r_{0}$.

(c) Masses of elementary particles may be hoped to follow from $r_{0}$ in a similar way as the term values of hydrogen from $h$ by way of the Rydberg constant.

(d) Interaction of particles provided the energy implied is of the order $h c / r_{0}$. This will be the case for the ordinary interaction in nuclei and for collision between particles rich in energy. Disintegration by 'explosion' may be expected if the nuclear field 'separates' from the nucleus on approaching another nucleus by less than $r_{0}$.

(e) Lengths less than $r_{0}$ will not be measurable, even by the $\gamma$-ray microscope, since the act of measuring would lead to nuclear explosions.

( $f$ ) A clue to the ultimate invariance property con. nected with $r_{0}$ will perhaps be obtained from relations between the observable values such as cross-sections for cosmic ray processes.

(3) R. A. Millikan discusses the "1938-values" of the fundamental constants. Acknowledging fully the importance of recent X-ray measurements by DuMond and Bollmann and by von Friesen, he arrives at :

$$
\begin{gathered}
e=4 \cdot 796 \pm 5 \times 10^{-10} \text { E.S.U., } h=6 \cdot 610 \pm 12 \times 10^{-27} \\
\text { erg. sec., } N=6 \cdot 097 \pm 6 \times 10^{23} .
\end{gathered}
$$

(4) Two papers deal with the influence of conditions imposed on the solutions of the Schrödinger equation. E. Schrödinger finds that $\psi$ need not necessarily be limited to single-valued functions in order to obtain unambiguous physical predictions. It is not possible, however, to have more than doublevalued functions, of opposite sign in the two branches, and the same problem admits of either mono- or divalent functions only for all its states.

A. Sommerfeld and $\mathbf{H}$. Welker surround an hydrogen atom by an infinitely high and steep spherical potential wall, and show that the ground state for this 'enclosed hydrogen atom' is expressed by con. fluent hypergeometric functions. With decreasing radius of the wall, the energy rises from its normal negative value and becomes positive when the available distance of the electron from the centre is limited to 1.835 times the normal ground state radius.

(5) Several papers are devoted to thermodynamical and statistical problems. W. Meissner goes into the details of the growth of solid carbon dioxide into liquid carbon dioxide as realized in the 'Agef ko' process. A very large heat gradient exists over a boundary layer of $0.1 \mathrm{~mm}$. in thickness which is traversed by capillaries $1 \mu$ in diameter. General equations for stationary thermodynamical processes at the moving boundary between two phases are given.

Statistical considerations initiated by Gibbs and Vollmer for problems of condensation are applied by $R$. Becker to the segregation of the constituents of a mixed crystal. The rate of formation of crystal nuclei for further growth can be determined as a 
function of under-cooling for a crystal in which the binding forces act between neighbouring atoms only. This compares well with the measurements.

M. v. Laue gives a study of the thermodynamics of supra-conductors, F. Hund the theory of diamagnetic properties of very small pieces of metal at low temperatures. The heat anomalies found for alums at very low temperatures by Simon and his co-workers are attributed to the internal electric and magnetic fields of the crystal acting on the trivalent metal ion by $\mathbf{P}$. Debye in a paper on cooling by adiabatic demagnetization. A detailed discussion of the iron-ammonium alum gives the Curie point at $0 \cdot 038^{\circ} \mathrm{K}$. as against the value $0.034^{\circ} \mathrm{K}$. given by
Simon from his observations. The magnetic coupling of the atoms leads to the formation of spontaneous order below a temperature of some thousandths of a degree, and prohibits a further cooling by demag. netization, which therefore will only be possible by action on the magnetic moments of the nuclei.

(6) Limitations of space forbid mention of further papers, save one by $K$. Schmeise and $W$. Bothe on a new kind of ultra-hard cosmic ray shower which seems to originate in the hard component of the primary cosmic rays. These showers scatter over an angle of about $10^{\circ}$, half that of normal showers, and are transformed into the ordinary soft showers on passing through matter.

\section{The Problem}

$\mathrm{T}$ HE problem of stellar energy was the subject of discussion of the Fourth Annual Conference on Theoretical Physics sponsored by the George Wash. ington University and the Carnegie Institution of Washington, and held in Washington, D.C., on March 21-23. The Conference was attended by astrophysicists studying the internal constitution of the stars (S. Chandrasekhar, B. Strömgren, T. Sterne, D. Menzel and others) as well as by physicists working on different branches of nuclear physics $(\mathbf{H}$. Bethe, G. Breit, G. Gamow, J. v. Neumann, E. Teller, M. Tuve, L. Hafstad, N. Heydenburg and others).

On the fundamental problem concerning the nuclear transformations as the source of energy of stars, some interesting conclusions have been reached. According to the so-called "aufbauhypothese", the process of the building up of the heavier elements from hydrogen is continually taking place in stellar interiors. Further, it is assumed that such processes liberate sufficient amounts of energy to account for the radiation of the stars. However, it seems that the model scheme proposed by von Weizsäcker $\left({ }_{2}^{4} \mathrm{He}+{ }_{1}^{1} \mathrm{H} \rightarrow{ }_{3}^{5} \mathrm{Li} \rightarrow{ }_{2}^{5} \mathrm{He}+\beta+\right)$ is contradicted by recent experimental evidence, according to which the nuclei $5 \mathrm{Li}$ and ${ }_{2}^{5} \mathrm{He}$ are both unstable, emitting heavy particles in a very short time. Another possible chain of reactions for the synthesis of the heavier elements from hydrogen and helium would require the stability of ${ }_{4}^{8} \mathrm{Be}$, which is again very doubtful according to recent experimental evidence. It appears, then, that the only course not excluded by present evidence on the binding energies of the light nuclei is the formation of ${ }_{4}^{6} \mathrm{Be}$ in triple collisions involving an $\alpha$-particle and two protons.

Although the existence of ${ }_{4}^{6} \mathrm{Be}$ has not yet been established experimentally, there are some indications that it might be a stable nucleus. If so, the chain of reactions leading to the synthesis of the heavier nuclei from hydrogen can be traced. The estimate of the probability of such reactions involving triple collisions shows that, under the conditions in the stellar interiors, the rate of liberation of energy will be sufficient to account for the radiation of the stars.

As another possibility the reaction ${ }_{1}^{1} \mathbf{H}+{ }_{1}^{1} \mathrm{H} \rightarrow$ ${ }_{1}^{2} \mathbf{H}+\beta+$ was suggested. It seems that the rate of such a reaction under the conditions in stellar interiors would be just enough to account for the radiation of the sun, though for stars much brighter than the sun other more effective sources of energy are required.

\section{Stellar Energy}

Special attention was directed to the possibility of resonance effects for nuclear reactions occurring in the interior of the stars; in such cases it would be necessary to consider that some light nuclei have virtual levels in the region of $10 \mathrm{kv}$. (corresponding to temperatures of the order $4 \times 10^{7}$ degrees Kelvin). The presence of resonance levels would not only increase the rate of liberation of energy of the reaction in question, but also would at the same time require a rather different type of stellar model. The rate of ordinary nuclear reactions increases exponentially with temperature, leading effectively to a point-source model in which the energy sources are concentrated at the centre. The selectivity with respect to tem. perature which would arise from resonance levels at about $10 \mathrm{kv}$. would, on the other hand, suggest a shell-source model with the maximum liberation of energy in a spherical shell surrounding the centre. It seems that this new model has a number of advantages over the point-source model.

The possibility of an extremely dense neutron core at the centre of the star (as proposed by L. Landau) was also discussed. The study of a number of known stars does not indicate a central condensation of more than what corresponds to 90 per cent of the total mass within half the radius. Thus, so far as astrophysical evidence is concerned, the model of a star with a heavy 'stellar nucleus' at the centre is not confirmed, except possibly for supergiants (for example, the $M$-component of vv Cephei, the infrared component of $\varepsilon$ Aurigæ), for which cases the observational evidence allows one to infer a high degree of central condensation corresponding to 90 per cent of the mass within less than ten per cent of the radius. Further, a stellar model with a 'nucleus' would require extremely high temperatures and densities $\left(\sim 16^{9}\right.$ degrees and $\left.\sim 10^{9} \mathrm{gm} . / \mathrm{cm} .{ }^{3}\right)$ at the surface of the core. In such physical circumstances all nuclear reactions would occur at extremely high rates and the total liberation of energy would lead to predicted luminosities for stars of altogether different orders of magnitude from those observed. It was therefore concluded that stellar models with a concentrated nuclear core cannot represent real stars.

\section{S. Chandrasekhar (Yerkes Observatory). \\ G. Gamow (George Washington University). \\ M. A. Tuve (Carnegie Institution of Washington).}

